Trauma Berufskrankh 2015 - 17[Suppl 1]:116-119 DOI 10.1007/s10039-013-2009-7

Online publiziert: 14. November 2013

(c) Springer-Verlag Berlin Heidelberg 2013
N. Drummer $\cdot$ R. Schmidt $\cdot$ F. Siemers

BG-Kliniken Bergmannstrost, Halle (Saale)

\title{
Arbeitsunfälle an der Hand
} Spezielle handtherapeutische Rehabilitation

\section{Entwicklung der speziellen handtherapeutischen Rehabilitation}

Auch nach fachgerechter und fachchirurgischer Versorgung von Handverletzungen, die sich berufsgenossenschaftlich versicherte Patienten im Rahmen von Arbeitsunfällen zuzogen, sind immer wieder lange Heilverläufe zu verzeichnen. Dauerschäden in rentenberechtigendem Ausmaß sind hierbei keine Seltenheit. Gründe für derartige unerfreuliche Verläufe sind u. a. Defizite in der postoperativen Rehabilitation. Die unzureichende Intensität der nachstationären Maßnahmen, fehlende Absprachen zwischen den am Rehabilitationsprozess beteiligten Berufsgruppen oder auch die fehlende Einbindung dringend erforderlicher Fachkompetenzen sind nur einige der Ursachen für mangelhafte Rehabilitationskonzepte.

Aus diesem Grund wurde in der Klinik für Plastische und Handchirurgie, Berufsgenossenschaftliche Kliniken Bergmannstrost, Halle (Saale), das Konzept der speziellen handtherapeutischen Rehabilitation 2001 etabliert und seitdem stetig weiterentwickelt.

Aktuell besteht die Kapazität für die stationäre Behandlung von 24 Patienten. Im Jahr 2012 wurden insgesamt 282 Patienten (+13\% gegenüber 2011) in der Klinik für Plastische und Handchirurgie, Berufsgenossenschaftliche Klinik Bergmannstrost, Halle (Saale), betreut. Hiervon konnten $68 \%$ in ihre letzte berufliche Tätigkeit reintegriert werden $(+12 \%$ gegenüber 2011). Bereits im 1. Quartal 2013 war ein weiterer Anstieg der Anzahl der betreuten Patienten um 13\% zu verzeichnen.

\section{Das Konzept - multimodal und interdisziplinär}

Im Mittelpunkt des multimodalen Behandlungskonzepts der speziellen handtherapeutischen Rehabilitation steht nicht nur das Zielorgan Hand, sondern der Patient in seiner Gesamtheit. Es sollen keine Spitzenleistungen ermöglicht, sondern die Funktionsfähigkeit für das alltägliche Leben und die zuletzt ausgeübte Tätigkeit trotz verbleibender Defizite wiedererlangt werden. Eine berufliche und soziale Reintegration wird angestrebt. Sollte dies nicht möglich sein, werden weitere Leistungen zur Teilhabe speziell am Arbeitsleben gebahnt.

In einem interdisziplinären Behandlungskonzept unter fachärztlicher handchirurgischer Leitung kann nicht nur eine notwendige weiterführende Diagnostik erfolgen, sondern es werden auch die physio-, ergo- sowie trainingstherapeutischen Therapieansätze und Testverfahren sowie der Übergang in die berufliche Wiedereingliederung vereint. Hierzu wird eine medizinische und berufsorientierte Rehabilitation angeboten.

Die meisten Therapeuten haben die Zusatzbezeichnung des Handtherapeuten über die Deutsche Arbeitsgemeinschaft für Handtherapie (DAHT) erworben.

\section{Organisation der Handrehabilitation}

\section{Medizinische handtherapeutische Rehabilitation}

Die stationäre Aufnahme in der speziellen Handrehabilitation findet grundsätzlich zu Beginn einer Arbeitswoche, an einem
Montag, statt. Im Vorfeld erfolgte die zur Durchführung der Maßnahme führende Planung über die Handsprechstunde der Klinik für Plastische und Handchirurgie, Berufsgenossenschaftliche Kliniken Bergmannstrost, Halle (Saale). Zum Teil handelt es sich um Patienten, die auf Veranlassung der jeweils zuständigen Berufsgenossenschaften im Rahmen einer Heilverlaufskontrolle (HVK) vorstellig werden, aber auch Patienten aus dem eigenen Verletztenkollektiv werden für die Durchführung der Handrehabilitation eingeplant. Ein Teil der HVK-Fälle werden im Zug des Modellprojekts Handchirurgie der DGUV (Deutsche Gesetzliche Unfallversicherung) an den Berufsgenossenschaftlichen Kliniken Bergmannstrost vorstellig.

Modellprojekt Handchirurgie der DGUV. Es wurde 2002 etabliert. Entsprechend des Rundschreibens der Landesverbände [1] sollte zur Verbesserung der handchirurgischen Versorgung bei schweren Handverletzungen spätestens 10 Tage nach einem Arbeitsunfall ein Handchirurg zugezogen werden, wenn noch Arbeitsunfähigkeit oder Behandlungsbedürftigkeit bestehen und davon ausgegangen werden kann, dass es sich um eine Verletzung mit handchirurgischem Problem handelt. Eine Vorstellung kann sich erübrigen, wenn der Durchgangsarzt selbst die Zusatzbezeichnung Handchirurgie besitzt.

Vereinzelt werden Patienten, die eine komplexe Handbehandlung benötigen, bereits im Rahmen der akutstationären Behandlung identifiziert.

Am Aufnahmetag erfolgt neben einer ausführlichen fachärztlichen klinischen 
Untersuchung des Patienten die Befunderhebung durch Physio- und Ergotherapeuten des Teams. Erste Bewegungsund Kraftmessungen werden durchgeführt und in den von der DGUV vorgegeben Messblättern festgehalten. Im Verlauf des Rehabilitationsprozesses werden diese Werte verlaufsabhängig regelmäßig reevaluiert.

An 5,5 Therapietagen in der Woche (Montag bis Samstags) findet neben physio- und ergotherapeutischen Einzeltherapien auch Werkstatttraining unter ergotherapeutischer Leitung statt. Zur allgemeinen Kräftigung und Beseitigung muskulärer Dysbalancen im Schultergürtel- und Wirbelsäulenbereich werden aus dem sporttherapeutischen Bereich die medizinische Trainingstherapie (MTT), allgemeiner Sport und Schwimmen mit integriert. Zusätzlich kommen semiaktive Anwendungen wie manuelle Therapie und Vojta-Therapie sowie passive Maßnahmen, z. B. Fango und Massagen, Narbenmassagen und Lymphdrainagen, zur Anwendung.

Insgesamt ist das komplette therapeutische Spektrum einer vollwertigen Rehabilitationseinrichtung verfügbar. Hilfsmittel und Trainingsgeräte (z. B. DigiFlex-Geräte, Kompressionshandschuhe) werden ausgetestet und angepasst. Die jeweils notwendigen Maßnahmen legt der betreuende Handchirurg fest.

Eine obligatorische psychologische Erstvorstellung erfolgt im Rahmen der Aufnahme des Patienten in die handtherapeutische Rehabilitation. Hier können neben der psychischen Stabilisierung, Unfallverarbeitung und der Vermittlung von Entspannungsverfahren auch unfallabhängige und -unabhängige Störungen abgegrenzt werden.

Durch die Schmerztherapeuten im Haus ist eine kontinuierliche Betreuung gewährleistet. Neben medikamentösen Therapien können Osteopathie, Entspannungsverfahren und auch invasive Maßnahmen, z. B. Stellatumblockaden, durchgeführt werden.

Zudem findet eine kontinuierliche Beratung und Betreuung durch unser Service- und Kompetenzzentrum für die DGUV statt. Falls notwendig können konsiliarisch weitere Fachdisziplinen einbezogen werden.
Einen sehr hohen Stellenwert im Gesamtkonzept der komplexen Handrehabilitation hat eine wöchentliche, jeweils Dienstags stattfindende interdisziplinäre Visite mit Physio- und Ergotherapeuten, Schmerztherapeuten, Psychologen und den Mitarbeitern des Service- und Kompetenzzentrums für die DGUV. Neben dem persönlichen Gespräch mit dem Patienten in Anwesenheit aller beteiligten Berufsgruppen werden auch die Messparameter objektiviert. Hierzu werden wöchentlich Bewegungsumfänge gemessen. Die Kraftentwicklung wird mit unserem computergestützten Jamar-Dynamometer eruiert. Es werden sowohl grobe als auch feine Griffformen ermittelt.

Zur weiterführenden Diagnostik können neben Nativröntgenaufnahmen, CT(Computertomographie) und MRT-Untersuchung (Magnetresonanztomographie) auch 3-Phasen-Szintigraphie und Thermographien zeitnah durchgeführt werden.

In den meisten Fällen wird das Rehabilitationsprogramm im Rahmen eines stationären Aufenthalts absolviert. Hierzu werden die Patienten in modern umgebauten 1- bis 2-Bett-Wohn-Einheiten in angrenzenden Wohnhäusern im Umfeld der Klinik untergebracht.

Das gesamte Rehabilitationsprogramm ist für einen Zeitraum von 3 Wochen ausgelegt. Im Vorfeld der Maßnahme wird ein entsprechender Kostenübernahmeantrag bei dem jeweiligen Kostenträger eingereicht. Er geht als Zwischenbericht mit einem aktuellen Befundbericht bei der zuständigen Berufsgenossenschaft ein. Der Sachbearbeiter soll dem Schreiben neben dem Rehabilitationspotenzial des Verletzten das spezifische Therapiekonzept sowie prognostische Einschätzungen zum Sachverhalt entnehmen können.

\section{Berufsorientierte handtherapeutische Rehabilitation}

Nach Abschluss der 3-wöchigen Komplexbehandlung kann in Einzelfällen die Aufnahme eines berufsorientierenden Trainings sinnvoll sein. Die Einleitung mit einer entsprechenden Beantragung der Kostenübernahme erfolgt kurzfristig in der Abschlusswoche der Handrehabilitation.
Im Rahmen des berufsorientierten Trainings, welches übergangslos nach der Handrehabilitation gestartet wird, findet eine Aufbelastung des Patienten berufsbezogen unter ärztlicher Leitung statt. Beginnend mit einer täglichen Belastung von $2 \mathrm{~h}$ wird im Verlauf auf $4 \mathrm{~h}$ pro Tag gesteigert. Die Betreuung erfolgt durch speziell ausgebildete Handwerker mit der Zusatzweiterbildung zur „Betreuung geistig und körperlich behinderter Menschen“.

$\mathrm{Zu}$ Beginn und Ende wird das Leistungsspektrum durch ein EFL-Screening (EFL: Evaluation der funktionellen Leistungsfähigkeit) evaluiert. Somit kann eine Aussage zum positiven und negativen Leistungsbild getroffen werden.

\section{Service- und Kompetenzzentrum für die DGUV}

Als einzigste Einrichtung für Handrehabilitation in Deutschland sind in den Berufsgenossenschaftlichen Kliniken Bergmannstrost die Berufsgenossenschaften in persönlicher Vertretung in der Einrichtung integriert. Das Service- und Kompetenzzentrum für die DGUV sorgt für die optimale Steuerung des Heilverlaufs, die direkt nach der operativen Versorgung beginnt und mit der Reintegration des $\mathrm{Pa}$ tienten endet.

Das Service- und Kompetenzzentrum übernimmt eine Mitverantwortung für die Durchführung, Sicherung, Überwachung und Entwicklung der Betreuungsqualität im Rahmen der medizinischen Rehabilitation. Es ist für die Beratung und ggf. Einleitung von Leistungen zur Teilhabe am Arbeitsleben sowie ergänzende Leistungen und die Zusammenarbeit mit anderen Berufsgruppen (Sozialdienst usw.) zuständig. Hierdurch wird die fachgerechte Durchführung einer patienten- und kostenträgerbezogenen Beratung (Einzelmaßnahmen) sowie zielorientierten Koordination und Steuerung von Behandlungsmaßnahmen im Rahmen des stationären Aufenthalts und bei der Entlassung insbesondere im Rehabilitationsbereich gesichert.

Im Rahmen der stationären Handrehabilitation haben die Patienten die Möglichkeit, sich neben der wöchentlichen Visite in Einzelgesprächen mit den Sach- 
bearbeitern des Service- und Kompetenzzentrums auszutauschen.

\section{Referenzzentrum für die Behandlung bei CRPS (,complex regional pain syndrome ${ }^{\prime \prime}$ )}

Das komplexe regionale Schmerzsyndrom (CPRS) stellt ein vielfältiges Krankheitsbild dar und erfordert ein ebenso breites Behandlungsspektrum.

Neben schmerzadaptierten rehabilitativen Maßnahmen werden Psychologen und Schmerztherapeuten engmaschig in das Behandlungsregime mit eingebunden. Hier kommen topische Behandlungsmaßnahmen, z. B. durch Schmerzpflaster oder topische Radikalfänger, aber auch systemische medikamentöse Therapien, z. B. Prednisolonstoßtherapie und WHOStufenschema-gerechte (WHO: „World Health Organization“) Schmerzmedikation, zur Anwendung. Bei schweren Verläufen ist kurzfristig eine stationäre Übernahme in die schmerztherapeutische Klinik des Hauses möglich.

\section{Standardisierte Berichterstattung}

Spätestens nach 3 Wochen wird dem Kostenträger eine standardisierte Berichterstattung zugeleitet. Hier werden neben der Entwicklung der Bewegungs- und Kraftumfänge auch die weitere bildgebende Diagnostik, Fotodokumentation und konsiliarische Befundung aufgezeigt.

Im Rahmen der Handrehabilitation arbeiten wir bereits seit vielen Jahren nach dem Ampelprinzip der ICD-F („International Statistical Classification of Diseases and Related Health Problems, function“). Dies sichert eine optimale Steuerung des Heilverfahrens.

\section{Rehabilitation von Anfang bis Ende}

Neben der medizinischen und berufsorientierten Rehabilitation sind bei Bedarf Fahrtrainings mit allen Fahrzeugtypen, Arbeitstherapie und arbeitstherapeutische Testungen sowie EFL-Testungen möglich.

Nach seiner Entlassung wird der $\mathrm{Pa}$ tient handchirurgisch im Rahmen von Arbeits- und Belastungserprobungen und weiteren ambulanten Nachkontrollen bis

Trauma Berufskrankh 2015 - 17[Suppl 1]:116-119 DOI 10.1007/s10039-013-2009-7

(c) Springer-Verlag Berlin Heidelberg 2013

\section{N. Drummer $\cdot$ R. Schmidt $\cdot$ F. Siemers}

Arbeitsunfälle an der Hand. Spezielle handtherapeutische Rehabilitation

\section{Zusammenfassung}

Hintergrund. Bei einer Handverletzung ist neben der adäquaten operativen Versorgung die postoperative konservative Therapie für die Wiedererlangung der Funktionsfähigkeit unerlässlich.

Handtherapeutische Rehabilitation. Die spezielle handtherapeutische Rehabilitation der Berufsgenossenschaftlichen Kliniken Bergmannstrost, Halle (Saale), ermöglicht den Einsatz des kompletten Spektrums einer Rehabilitationseinrichtung und der Unterstützung einer Klinik der Maximalversorgung. Die einzigartige Integration des Service- und Kompetenzzentrums für die DGUV (Deutsche Gesetzliche Unfallversicherung) in die Klinik sichert die optimale Koordinierung des Heil- verfahrens, Beratung des Patienten und frühzeitige Einleitung weiterer Leistungen. Resümee. In einem multimodalen, interdisziplinären Behandlungskonzept unter fachärztlicher handchirurgischer Leitung und Einbeziehung von Psychologen und Schmerztherapeuten kann eine bestmöglichste Versorgung und schnellstmögliche Reintegration des Patienten in das Berufs- und Sozialleben gewährleistet werden.

\section{Schlüsselwörter}

Handchirurgie - Rehabilitation · Komplexes regionales Schmerzsyndrom (CRPS) . Handverletzung · Heilverfahren

\section{Work-associated injuries to the hand. Special therapeutic rehabilitation for the hand}

\section{Abstract}

Background. With a hand injury, adequate surgical treatment and postoperative conservative treatment are both essential to recover function.

Therapeutic rehabilitation for the hand. Special therapeutic rehabilitation for the hand at the professional cooperative clinics Bergmannstrost uses of the complete spectrum of the rehabilitation facility and the support of a maximum care hospital. The unique integration of the service and competence center in the clinic ensures optimal coordination of the healing process, patient counseling and early initiation of further services.
Conclusion. Using a multimodal, multidisciplinary treatment approach under the medical supervision of hand surgeons and involvement of psychologists and pain specialists, the best possible care and the fastest possible reintegration of the patient into professional and social life is guaranteed.

\section{Keywords}

Hand injuries, surgery · Rehabilitation · CRPS (complex regional pain syndromes) $\cdot$ Hand injuries $\cdot$ Remedies zum Abschluss des Heilverfahrens und ggf. Begutachtung weiterbetreut.

Zusätzlich besteht die Möglichkeit stationärer Heilverlaufskontrollen sowie der Durchführung von Ergo-Physio-Konsultationen direkt am Arbeitsplatz.

Zudem können nach Amputationen Prothesen, auch unter Einbeziehung heimatnaher Orthopädietechniker, angepasst werden. Eine Prothesenaustestung wurde standardisiert etabliert.

\section{Fazit für die Praxis}

- Die spezielle handtherapeutische Rehabilitation der BG-Klinik Bergmannstrost sichert nach einer Handverletzung unter fachärztlich-handchirurgischer Leitung durch ein intensives physio-, ergo- sowie trainingstherapeutisches Komplexprogramm, welches auf die individuellen Bedürfnisse des handverletzten Patienten abgestimmt wird, einen ununterbrochenen Behandlungsverlauf mit nachweislich erfolgreichem Outcome bei einer Vielzahl der Betroffenen. 
- Das multimodale Behandlungskonzept ermöglicht eine allumfassende fachkundige Betreuung des Patienten aus einer Hand. Eine Betreuung von der Akutversorgung bis zur beruflichen und sozialen Reintegration ist möglich.

- Durch die Integration der Rehabilitation in eine Klinik der Maximalversorgung kann ein Optimum der Patientenversorgung in allen Bereichen erreicht werden. Das komplette Spektrum der berufsgenossenschaftlichen Anforderungen im Heilverfahren kann abgebildet werden.

- Mit dem interdisziplinären Behandlungsregime ist eine schnellstmögliche Rückführung des Patienten in das Alltags- und Berufsleben gewährleistet. Eine Minderung der verbleibenden MdE (Minderung der Erwerbsfähigkeit) und Reduktion der ökonomischen Belastung ist hierdurch möglich.

\section{Korrespondenzadresse}

Dr. N. Drummer

BG-Kliniken Bergmannstrost,

Merseburger Straße 165, 06112 Halle (Saale)

Nicole.Drummer@Bergmannstrost.com

\section{Einhaltung ethischer Richtlinien}

Interessenkonflikt. N. Drummer, R. Schmidt und F. Siemers geben an, dass kein Interessenkonflikt besteht.

Dieser Beitrag beinhaltet keine Studien an Menschen oder Tieren.

The supplement containing this article is not sponsored by industry.

\section{Literatur}

1. NN (2005) Rundschreiben H3/2005 Modellprojekt „Verbesserung der handchirurgischen Versorgung". LV NWD, H3/2005 vom 22.02.2005. Dok.-

Nr.: 412.36-Modellprojekt 